\title{
Über die hohe Lebenserwartung der japanischen Bevölkerung - Faktoren und mögliche Ursachen
}

\section{Einleitung - Begriffsdefinitionen}

Die Lebenserwartung misst das durchschnittliche Sterbealter einer Bevölkerung und wird mithilfe demografischer Kennzahlen, der Geburten- und Sterberate, ermittelt [1]. Die Geburtenrate erfasst als statistische Kennzahl die Lebendgeborenen innerhalb eines Jahres bezogen auf die Gesamtbevölkerung des Landes. Die Mortalitätsrate, ebenso eine wichtige Kennzahl der Demografie, gibt die Anzahl der Todesfälle in Bezug auf die Einwohnerzahl an. Die Geburtenrate der japanischen Bevölkerung lag im Jahr 2018 bei etwa 7,5 und die Sterberate bei 9,9 pro 1000 Einwohner [2].

Gemäß den Angaben der Weltgesundheitsorganisation (WHO) kann ein Mädchen, das 2018 in Japan geboren wurde, mit einer Lebenserwartung von 87 Jahren rechnen, ein Junge mit 81,5 [3]. Ganz anders präsentierten sich die Daten zu Beginn des 20. Jahrhunderts. Im Zeitraum von 1917-1923 lag die Sterberate der Japaner besonders hoch und erreichte 1918 ein Maximum von 27,3. Die damals schlechten Lebensverhältnisse, die mangelhafte medizinische Versorgung, Hygiene und Infrastruktur mochten als Gründe dafür verantwortlich gewesen sein. $A b$ diesem Zeitpunkt begann die Sterberate, abgesehen von kleinen Schwankungen, $\mathrm{zu}$ sinken. Insgesamt betrachtet lag das Minimum der Sterberate im Jahr 1980 bei ungefähr 6,0. Danach stieg jedoch die Ster-

Hinweis der Redaktion: Dieser Artikel entstammt einer vorwissenschaftlichen schulischen Arbeit von Leonhard Kostner (BORG1, Hegelgasse 12, 1010 Wien) in gekürzter Form.

Deutsche Zeitschrift für Akupunktur $2021 \cdot 64$ (4): $280-282$ https://doi.org/10.1007/s42212-021-00420-6 Angenommen: 3. September 2021 Online publiziert: 1. Oktober 2021 (c) Springer Medizin Verlag $\mathrm{GmbH}$, ein Teil von Springer Nature 2021 berate wieder langsam an, da ab diesem Zeitpunkt der Anteil älterer Menschen innerhalb der Bevölkerung schon relativ hoch war [4].

Im Vergleich zur EU sind die Daten zur Kindersterblichkeit in Japan niedriger. Die Kindersterblichkeit, das heißt die Wahrscheinlichkeit eines Kindes, vor dem 5. Lebensjahr zu sterben bezogen auf 1000 Lebendgeburten, liegt in Japan bei 2,5, in der EU betrug sie 2018 4,1 [5].

Alle diese Daten sind bewusst noch vor der Pandemie liegend gewählt worden, die demografischen Daten aus 2020 (zur Ergänzung auch ein Wert aus 2016) finden sich in Tab. 1 und 2 [6-9].

Statistische Daten differieren, je nachdem, welche Quelle man heranzieht. Wirtschaftskammer, WHO, Central Intelligence Agency (CIA) und viele andere Organisationen veröffentlichen regelmäBig diverse demografische Daten. In der Tabelle der durchschnittlichen Lebenserwartung der Weltgesundheitsorganisation WHO führt derzeit Japan mit 84,3 Jahren [9]. Welche Faktoren könnten für die lange Lebensdauer verantwortlich zeichnen?

\section{Gesunde Ernährung}

Das traditionelle, japanische Gericht besteht aus einer Schale Reis (Gohan) und mehreren kleinen Beilagen (Okazu; [10]). Reis stellt das Grundnahrungsmittel dar und wird zu allen 3 Hauptmahlzeiten serviert. In Verwendung sind 2 Arten: süßer Naturreis (Mochigome) und weißer Reis (Uruchimai). Studien zeigen, dass eine häufige Reisnahrung mit einer Zunahme von Diabetes 2 einhergeht [11]. Dies wird vor allem dem weißen Reis zugeschrieben, Naturreis und ungeschälter oder halb geschälter Reis könnten hier Abhilfe schaffen.

Aufgrund der geografischen Lage ist der Verzehr von Fisch in Japan weit verbreitet.

Bereits seit dem 17. Jahrhundert wird in Japan roher Fisch, Sashimi, gegessen
[12]. Zunehmend gerät auch der einst als "gesund" erachtete Fisch in den Fokus der Medien. Mikroplastik, Quecksilber, Pflanzenschutzmittel ohne gesetzlich festgelegte Grenzwerte und Parasiten stellen nur eine kleine Auswahl dar, weshalb bei Fisch besonders auf die Herkunft geachtet werden sollte $[13,14]$. Auch die im Fisch reichlich vorhandenen Omega3-Fettsäuren, die gemeinhin als kardiovaskulär protektiv angesehen werden, sorgen für kritische Diskussionen in kürzlich erschienenen Metaanalysen [15].

Anders sieht es mit fermentierten Lebensmitteln aus. Studien belegen den positiven Effekt auf die Gesundheit, eine Veröffentlichung der Universität Leipzig beschreibt den molekularen Mechanismus, wie Bakterien aus fermentierten Nahrungsmitteln mit dem menschlichen Immunsystem interagieren [16].

Miso, fermentierte Sojapaste, und Natto, fermentierte Sojabohnen, stehen häufig auf dem japanischen Speiseplan. Hinzu kommen unfermentierte Sojaprodukte, wie beispielsweise Tofu, welche zur Vorbeugung von chronischen Herzerkrankungen beitragen können [17].

Der in Japan viel getrunkene Grüntee gilt als besonders gesundheitsfördernd. Das im grünen Tee reichlich vorhandene Epigallocatechin-3-gallat soll nicht nur das Wachstum von Krebszellen behindern, neben der antioxidativen Wirksamkeit ließ sich in vitro ebenso eine ausgeprägte antivirale und antibakterielle Wirkung nachweisen [18-20].

Die Präfektur Okinawa weist verglichen mit vielen anderen Ortschaften Japans einen besonders hohen Anteil älterer Menschen innerhalb der Bevölkerung auf. Möglicherweise zeichnet die besonders kalorienarme und traditionelle Ernährungsweise der älteren Generation dafür verantwortlich. Jedoch sinkt die Lebenserwartung der jüngeren Generation, vielleicht weil die traditionelle japanische 
Tab. 1 Lebenserwartung (LE) für Neugeborene 2020 im Vergleich [2, 6, 7, 9]

\begin{tabular}{|l|l|l|l}
\hline Land & LE Frauen & LE Männer & LE gesamt \\
\hline EU & 81,3 & 75,1 & 78,2 \\
\hline Japan & 86,9 & 81,5 & 84,3 \\
\hline Welt & 75,0 & 70,6 & 73,3 \\
\hline Österreich & 84,2 & 79,5 & 81,6 \\
\hline Deutschland & 84,8 & 78,7 & 81,7 \\
\hline Schweiz & 85,1 & 81,8 & 83,4 \\
\hline
\end{tabular}

Tab. 2 Geburten- und Sterberate im Vergleich [8]

\begin{tabular}{|l|l|l}
\hline Land/Jahr & Geburtenrate & Sterberate \\
\hline EU/2016 & 10,1 & 10,2 \\
\hline Japan/2020 & 7,32 & 10,19 \\
\hline Welt/2020 & 18,1 & 7,7 \\
\hline Österreich/2020 & 9,5 & 9,82 \\
\hline Deutschland/2020 & 8,62 & 12,1 \\
\hline Schweiz/2020 & 10,45 & 8,5 \\
\hline
\end{tabular}

Küche vom amerikanischen Fastfood verdrängt wird [21].

\section{Hygiene und Gesundheitspflege}

Aufgrund der alternden Bevölkerung und der steigenden Lebenserwartung sind die Japaner immer mehr auf gegenseitige Hilfe angewiesen. Die tägliche Körperhygiene und eine gute medizinische Versorgung sind wichtige Voraussetzungen für ein gesundes und langes Leben. Die Dichte der Bevölkerung, vor allem in Städten wie Tokio, begünstigt die schnelle Verbreitung von Infektionskrankheiten. Da die japanische Bevölkerung jedoch sehr viel Wert auf Hygiene und Abstand legt, können sich Infektionskrankheiten weniger leicht ausbreiten als anderswo - so auch zu Zeiten der COVID-19-Pandemie.

Ein wichtiger Bestandteil der Gesundheitspflege in Japan ist das regelmäßige Baden in sogenannten Furos. Das traditionelle Baden in heißem Wasser (bis $42{ }^{\circ} \mathrm{C}$ ) sorgt nicht nur für die Reinigung des Körpers, sondern dient auch der Entspannung. $80 \%$ der unter 40 -jährigen Japaner und Japanerinnen genießen täglich ihr heißes Bad. Beliebt ist auch der Besuch öffentlicher Thermalbäder (Onsen). Diese natürlichen heißen Quellen enthalten viele Mineralien, welche sogar als Badezusatz zu Hause Verwendung finden. Der Gesundheitseffekt der Hyperthermie und der Badezusätze, verbunden mit der traditionellen japanischen Badekultur, konn- te in einer Studie nachgewiesen werden. Anhand der Auswertung verschiedenster Parameter wie Blutbild, pH-Wert, Gewichtsverhalten, Körpertemperatur kommen die Autoren zu dem Schluss, dass traditionelles Baden die Gesundheit und die Langlebigkeit fördert [22].

\section{》) Japan verfügt über ein modernes Gesundheits-} system mit hochmoderner medizinischer Infra-

\section{struktur}

Japan verfügt über ein modernes Gesundheitssystem mit hochmoderner medizinischer Infrastruktur, vor allem in den Ballungszentren. Einige weltweit agierende Pharmakonzerne und Medizintechnikunternehmen haben ihren Hauptsitz in Japan. Verglichen mit Deutschland weist Japan zwar eine geringere Anzahl an Ärzten pro Einwohner auf, jedoch eine relativ hohe Anzahl an Spitalsbetten. Daraus resultiert, dass längere Krankenhausaufenthalte möglich sind bzw. schneller eine stationäre Therapie beginnen kann [23].

Eine Besonderheit am japanischen Gesundheitssystem besteht auch in der Integration traditioneller Arzneithera- pie. Kampo-Medizin wird bei Erkältungskrankheiten, aber auch bei vielen chronischen Erkrankungen von Ärzten gerne verordnet und die Kosten werden von der Krankenkasse übernommen [24].

Ikigai

In Japan herrscht die Meinung, dass die richtige Lebenseinstellung und eine positive Denkweise das Leben verlängern können. Das Wort Ikigai beschreibt den Sinn des Lebens beziehungsweise den Grund, wofür es sich zu leben lohnt. Dieses Gefühl ist mehr mit der Zukunft als mit der Gegenwart verbunden und soll stets ein Ziel vermitteln, welches die Menschen dazu antreibt, ihren Lebensweg trotz schlechter Zeiten weiterzugehen und eine positive Geisteshaltung zu bewahren [25]. Andererseits meint Ikigai auch die Beschäftigung mit Dingen, die das Leben lebenswert machen, die Entwicklung der eigenen Kreativität, sich auch im Alter nicht nutzlos fühlen, bis zuletzt ein sinnzentriertes Leben zu führen. All das und auch das Gefühl, zu einer Gruppe zu gehören, könnte die Resilienz besonders bei Älteren stärken und zu einer Lebensverlängerung führen. In der "Ohsaki-Studie“ befragten die Autoren 43.391 erwachsene Japaner: „Verspüren Sie Ikigai in Ihrem Leben?" 7 Jahre später wurden die Ergebnisse ausgewertet. 3048 Studienteilnehmer verstarben während dieser Zeit. Das Gesamtmortalitätsrisiko war signifikant höher bei den Probanden, denen Ikigai nichts bedeutete, im Vergleich mit den Personen, die sehr wohl Ikigai verspürten. Das Risiko, an kardiovaskulären Erkrankungen zu versterben, wies einen sehr deutlichen Unterschied auf, wohingegen die Chance, an einem Karzinom zu versterben, in beiden Gruppen gleich ausfiel [26].

Obwohl Ikigai schon einige hundert Jahre bekannt sein dürfte, erschienen erst Mitte des 20. Jahrhunderts genauere Angaben. Als „Mutter von Ikigai“ wird die Psychiaterin Mieko Kamiya bezeichnet, die ein bis dato nur in japanischer Sprache zu lesendes Werk über Ikigai publizierte $[27,28]$. Ihr zufolge besteht das Ikigai aus 7 Grundbedürfnissen:

- das Bedürfnis nach einer erfüllenden Existenz,

- das Bedürfnis nach Wachstum und Veränderung,

- positive Zukunftsaussichten,

- das Verlangen nach Antworten, 
- das Bedürfnis nach Selbstverwirklichung,

- das Streben nach Freiheit,

- die Motivation zur Selbstverwirklichung.

\section{Fazit}

Die Lebenserwartung der japanischen Bevölkerung gehört zu den höchsten der Welt und beträgt derzeit etwa 84 Jahre, Japanerinnen leben beinahe 87 Jahre. Das modern ausgebaute Gesundheitswesen, eine gute Infrastruktur, die stabile politische Lage, das gemäßigte Klima, die hohen Hygienestandards und die ausgewogene Ernährung tragen sehr wahrscheinlich zur hohen Lebenserwartung der japanischen Bevölkerung bei.

Literatur

1. Roser M (2019) Ourworld in data - Life expectancy, Oxford University. https://ourworldindata.org/ life-expectancy. Zugegriffen: 12. Okt. 2019

2. WKO - Wirtschaftskammer Österreich (2019) Geburten- und Sterberaten, Österreich. http://wko.at/ statistikleu/europa-geburtenrate.pdf. Zugegriffen: 29. Juni 2021

3. WHO Life expectancy and healthy life expectancy. https://apps.who.int/gho/data/node.main.688. Zugegriffen: 28. Juni 2021

4. Ministry of Health, Labour and Wellfare (2005) The 20th Life table 1900-2004, Japan

5. World Bank (2018) Mortality rate, under-5 (per 1,000 live births)_Japan, England. https://data.worldbank.org/indicator/SH.DYN. MORT?locations=JP. Zugegriffen: 23. Dez. 2019

6. https://www.laenderdaten.info/lebenserwartung. php\#by-population. Zugegriffen: 23. Juli 2021

7. https://www.statistik.at/web_de/statistiken/ menschen_und_gesellschaft/bevoelkerung/demographische_indikatoren/index.html. Zugegriffen: 12. Juli 2021

8. https://WWw.indexmundi.com $/ \mathrm{g} / \mathrm{g}$. aspx? $v=25 \& c=$ sz\&l=de. Zugegriffen: 17. Aug. 2021

9. https://en.wikipedia.org/wiki/List_of_countries_ by_life_expectancy\#List_by_the_World_Health_ Organization_(2020).ZZugegriffen: 29. Juni 2021

10. Stimac M (2002) Essen Japaner gesünder?, Deutschland. https://japanlink.de/landleute/essentrinken/essen-japaner-gesuender/. Zugegriffen: 24. Okt. 2019

11. Hu EA, Pan A, Malik V, Sun Q (2012) White rice consumption and risk of type 2 diabetes: metaanalysis and systematic review. BMJ 344:e1454

12. Ishige N (1990) The cambridge world history of food-Japan, Japan. https://www.cambridge. org/ph/files/8614/1234/6051/Cambridge-World-
History-Of-Food-VB4-Japan.pdf. Zugegriffen: 31. Okt. 2019

13. Carmo J, Marques S, Bispo M, Serra D (2017) Anisakiasis: a growing cause of abdominal pain! BMJ Case Rep. https://doi.org/10.1136/bcr-2016218857

14. https://www.peta.de/veganleben/so-schaedlichist-fischkonsum-fuer-die-gesundheit/. Zugegriffen: 16. Juli 2021

15. Aung T, Halsey J, Kromhout D et al (2018) Associations of omega-3 fatty acid supplement use with cardiovascular disease risks. JAMA Cardiol 3(3):225-233. https://doi.org/10.1001/jamacardio.2017.5205 (Meta-analysis of 10 Trials Involving 77917 Individuals)

16. Peters A, Krumbholz P, Jäger E, Heintz-Buschart A, Çakir MV et al (2019) Metabolites of lactic acid bacteria present in fermented foods are highly potent agonists of human hydroxycarboxylic acid receptor. PLoS Genet. https://doi.org/10.1371/journal.pgen. 1008145

17. Nozue M, Shimazu T (2017) Fermented soy product intake is inversely associated with the development of high blood pressure: the Japan public health center-Based prospective study, Oxford. https://academic.oup.com/jn/article/147/9/1749/4743538. Zugegriffen: 31. Okt. 2019

18. Saeki K, Minami T, Isemura M (2017) Health benefits of green tea: an evidencebased. Approach, Japan, S 29-31

19. Fujiki H (2002) Cancer letters - Green tea: cancer preventive beverage and/or drug, Ireland. https:// wWw.sciencedirect.com/science/article/pii/ S0304383502003798. Zugegriffen: 1. Nov. 2019

20. Steinmann J, Buer J, Pietschmann T, Steinmann E (2013) Anti-infective properties of epigallocatechin-3-gallate (EGCG), a component of green tea. Br J Pharmacol 168(5):1059-1073. https:// doi.org/10.1111/bph. 12009

21. Gavrilova N, Gavrilov L (2001) Comments on dietary restriction, okinawa diet and longevity, Chicago. https://www.karger.com/Article/Pdf/329894. Zugegriffen: 23. Dez. 2019

22. Watanabe M, Koboshi S, Yoshimoto H (2016) Traditional Japanese style bathing may contribute to good health and longevity, Japan. https://WwW. scirp.org/journal/PaperInformation.aspx?PaperlD= 67011\&\#abstract. Zugegriffen: 5. Dez. 2019

23. Fukawa T (2002) Public health insurance in Japan, Japan. http://citeseerx.ist.psu.edu/viewdoc/down load?doi=10.1.1.843.6277\&rep=rep 1\&type $=$ pdf. Zugegriffen: 5. Dez. 2019

24. Takahashi T, Moriya J (2006) Traditional Chinese medicine and Kampo: a review from the distant past for the future, Japan https:// doi.org/10.1177/147323000603400301

25. Nakanishi N (1999) 'Ikigai' in older Japanese people. Age Aging 28:323-324
26. Toshimasa S, Nakaya N, Ohmori K, Shimazu T, Mizuka H (2008) Sense of life worth living (Ikigai) and mortality in Japan: Ohsaki study. Psychosom Med 70(6):709-715. https://doi.org/10.1097/ PSY.06013e31817e7e64

27. MiekoK(1966) Ikigainitsuite (生きがいについて; engl. About Ikigai). Misuzu Shobō, Tōkyō

28. https://de.wikipedia.org/wiki/lkigai. Zugegriffen: 23. Juli 2021

Korrespondenzadresse

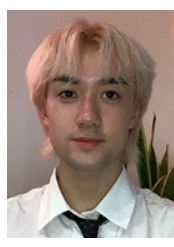

Leonhard Kostner Hochstraße 4, 1230 Wien, Österreich

\section{Einhaltung ethischer Richtlinien}

Interessenkonflikt. L. Kostner gibt an, dass kein Interessenkonflikt besteht.

Für diesen Beitrag wurden von den Autoren keine Studien an Menschen oder Tieren durchgeführt. Für die aufgeführten Studien gelten die jeweils dort angegebenen ethischen Richtlinien. 